Historic, Archive Document

Do not assume content reflects current scientific knowledge, policies, or practices. 

Albania-Pure glistening white. Wide open. Ruffled

Alice Tiplady (P)-Orange-saffron. Large flowers

Altair (P)-Salmon-saffron. Extra tall.

Alton-Bright orange. Ruffled. Strong grower

America-Soft lavender pink. Large flowers

Anita (P) - Rich canary yellow.

Anna Eberius-Deep velvety purple. Darker throat

Arden (P) - Watermelon red. Ruffled. Tall. Graceful

Arizona Rose-Rose pink. Nearly a solid color

Arlon (P) - Light salmon. Beautiful red throat

Ashburn-Salmon red, flaked darker. Very large

Atherton-Deep salmon. Petals clearly blue bordered

Baron Hulot-Indigo blue. Fine golden throat lines

Beaconflame-Flame scarlet. Strong grower

Bertrex - White. Lilac markings in throat.

Blackhawk-Cardinal red, much darker center

Bobolink (P)-Orange, tinted yellow. Tall. Slender

Bordeaux-Deep wine color. Large flowers.

Butterboy (P)-Butter - $u$ p yellow. Large. Vigorous

Butterfly (P)-Pale salmon yellow. Ruffled

Byron L. Smith-Lavender pink. Pale yellow throat

Canopus (P) - Rich solid yellow. Large flowers

Capella (P) - Fiery orange red. Large flowers. Tall

Cardisun-Dark red, darker throat. Strong grower

Carmen Sylva-Pure white. Throat penciled lilac

Challenger-Solid rich velvety red. Strong. Vigorous

Chateau Thierry-Orange red. Deep red blotch. Large

Chicago White-Creamy white. Rose markings in throat

Clio (P)-Orange. Deep yellow throat. Tali. Slender

Crescent Queen-Dark wine red. Ruffled. Large

Crimson Glow-Scarlet. Fine yellow markings. Large

Diana-Bright red. Large wide open flowers. Tall

Dixie-Deep rich red. Large flowers.

Dorothy McKibbin-Rose pink. Lighter throat. Tall

Dorothy Wheeler (P)-Delicate rose pink. Yellow throat

Dorrit-Lavender pink, large flowers. Tall. Strong

Duchess of York-Purple blue. Large. Tall. Straight

Eda (P) -Deep salmon buff. Large flowers. Tall

E. J. Shaylor-Pure deep rose pink. Large. Ruffled

Elberton (P) - Very clear creamy yellow. Tall

Elf-Pure white. Pale yellow throat. Perfect spike

Elizabeth Tabor-Rose pink. Crimson blotch. Large

Elora-Creamy white. Pink and cream throat. Tall

Evelyn Kirtland-Rose pink. Scarlet blotch. Very tall

Fair Columbian-Lilac. Deeper throat. Tall

Fairest White-Pure white. Large flowers. Fine form

Fairfax-Solid magenta. Very large and strong

Flaming Vale-Deep cardinal red. Tall and strong

Firefly (P) -Extra bright deep scarlet

Flora-Pure yellow. Large flowers. Tall, strong plant

Floral Treasure-Deep lavender pink. Straight. Tall

Frank J. Symmes-Peach red. Ruffled. Very vigorous

Giant Fawn-Deep fawn yellow. Large flowers

Giant Myrtle-Soft pink. Large, wide open flowers

Giant Nymph-Rose pink. Creamy throat. Very large

Glendale-Dark rose red. Very tall and strong

Gold-Pure golden yellow. Large flowers. Fine form

Golden Butterfly (P)-Deep canary yellow. Graceful

Golden Glory-Primrose yellow. Ruffled. Strong

Golden Measure-Pure yellow, no markings. Very tall

Goliath-Pansy purple. Lighter throat. Vigorous

Governor Hanley-Cardinal red. Large flowers. Tall

Gretchen Zang-Soft pink. Scarlet throat. Tall. Strong

Halley-Salmon. Yellow in throat. Tall and vigorous

Herada-Clear mauve. Large flowers. Tall

Ida Van-Deep salmon red. Large flowers

Illuminator-Brilliant carmine, tinted blue. Very tall

Jacoba Van Beieren-Pure violet self color. Large

Jenny Lind-Apricot pink. Soft yellow throat

Jewell (P) - Light salmon pink. Golden throat. Wide open

Joe Coleman-Rich red. Ruffled. Strong

Kennemerland-True rose color. Purple throat. Strong

Le Marechal Foch-Pale pink. Very large flowers

Lilac Glory-Soft lilac rose pink. Ruffled. Strong*

Lilac Old Rose (P) - Old rose. Large and tall

Lilywhite-Pure creamy white. Slight throat markings

Louise-Lavender. Light purple blotch. Large flower

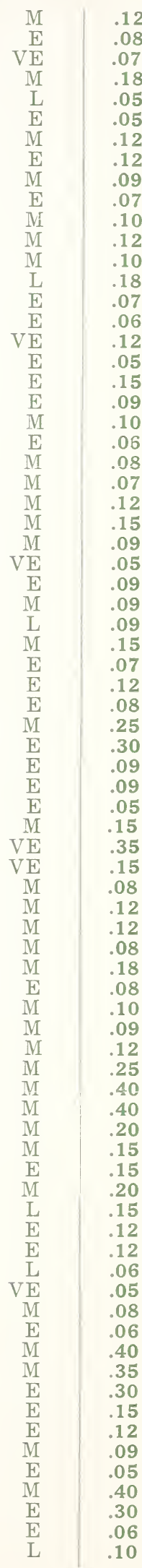


Loveliness-Pale cream. Beautifully formed flowers

Magic-Pale lavender blue. Deep purple blotch. Tall

Maiden's Blush (P) -Delicate pink. Lighter throat Maine-Pure glistening white. Tall and straight

Marie Kunderd-Purest white. Ruffled. Extra choice Marietta-Light salmon, shading to burnt orange

Mary Fennell-Deep lilac. Primrose yellow throat

Mary Pickford-Creamy white. Sulphur yellow throat

Mary Stearns Burke-Canary yellow, overlaid apricot

Masterpiece-Rose red. Large ruffled. Tall

Master Wietze-Dark violet. Tall. Slender.

Midsummer Dream (P) - Geranium red. Large flowers

Ming Toy (P)_Deep buff yellow. Large flowers. Tall

Mona Lisa-Pale rose pink. Large ruffled flowers

Mrs. Dr. Norton-Cream and pink. Tall and slender

Mrs. F. C. Peters-Rosy lilac. Soft crimson blotch

Mrs. Francis King-Vermilion red. Very large. Tall

Mrs. Frank Pendleton-Rose pink. Large deep red blotch

Mrs. H. E. Bothin-Flesh pink, scarlet center. Ruffled

Mrs. Watt- "American Beauty" red. Strong grower

Mrs. Wm. Kent-Shrimp pink, striped rose. Large. Many open

Myra (P) -Deep salmon on yellow ground. Large. Tall

Myrtle-Delicate pink, blending to nearly white throat

Neoga-Garnet red. Slightly ruffled. Strong

Niagara-Pale yellow, penciled carmine. Very strong

1910 Rose-Pure rose pink. Large flowers. Tall plant

Odin-Jasper red. Rose blotch. Large, wide open

Orange Glory-Orange, lighter throat. Ruffied. Strong

Orange Queen (P)-Apricot orange. Large and tall

Panama-Rich rose pink. Large flowers. Strong grower

Peace-White, rose throat markings. Very tall and strong

Peach Rose-Deep rose pink. Large flowers

Pink Beauty-Rose pink. Blood red blotch.

Pink Lily-Deep rose pink. Ruffled

Pink Perfection-Soft pink. Large flowers. Graceful

Pink Wonder-Pale pink. Light yellow throat. Very large

Pollyanna-Clear golden yellow. Ruffled.

Prince of Wales-Salmon-rose pink. Yellow and red throat

Purple Glory-Deepest maroon. Almost black blotch

Pythia-Solid spectrum red. Extra large flowers

Rajah-Dark red. Very large flowers. Tall

Rev. Ewbank-Porcelain blue. Slender and graceful

Roanoke (P) - Rich yellow. Vigorous

Romance-Orange salmon, bordered wine blue

Rosalia (P) -Yellow and orange. Ruffled

Rose Ash-Ashes of roses. Spike tall and very straight

Rosella-Light rose. White throat. Large

Rose Glory-Pure rose pink. Large ruffled flowers

Rose Wells-Pale rose. Orchid throat. Large. Tall

Rouge Torch-Creamy white. Intense crimson throat

Salmon Beauty (P) -Deep salmon. Yellow throat

Scarlano-Bright orange red. Ruffled

Scarlet Princeps-Solid scarlet. Throat deeper

Scarlet Wonder-Intense scarlet. Very large and tall

Schwaben-Canary yellow. Large and very strong

Sentinel-Pure rose pink. Tall

Shell Pink (P)-Delicate pink. Lighter throat

Sheila (P) - Light coral red. Flesh pink throat. Large

Sirius (P)-Dark salmon. Yellow throat. Ruffled

Sonia (P) -Bright red. Large and tall

Souvenir (P)-Pure golden yellow. Large. Vigorous

Taro- "American Beauty" rose. Very tall and strong

Tempa-Old rose and Pink. Bronze throat. Ruffled

Thoth (P) - White, canary yellow throat. Tall

Tiffany-Pure white. Slightly ruffled. Vigorous

Topaz (P) - Salmon pink and buff. Graceful

Twinkles (P) - Salmon rose. Tall and slender

Tyrian Beauty-Rose red. Very tall and strong

Utopia (P)-Orange salmon, yellow throat. Large

Violet Glory-Rich deep violet self color. Ruffled

War-Pure red. Large and tall

White Butterfly (P)-White, creamy throat. Tall

White Glory-White. Iris blue throat. Ruffled

White Wonder-Pure white. Very large. Wide open

Wilbrink-Pale pink. Yellow in throat

Yellow Hammer-Yellow. Red throat markings. Tall

Zenobia ( $P$ )_-Orange red. Tall and slender

M

$\mathrm{VE}$

$\mathrm{VE}$

$\underset{\mathrm{VE}}{\mathrm{E}}$

M

E

N

$M$
$M$

N

$\mathrm{M}$

$\stackrel{E}{M}$

M

$\stackrel{\mathrm{L}}{\mathrm{M}}$

$\mathrm{M}$
$\mathrm{M}$

M

M

M

E

$\mathrm{E}$

$\stackrel{\mathrm{E}}{\mathrm{M}}$

M

M

$\mathrm{E}$

M

E

VL

VE

M

M

M

E

M

$\mathrm{M}$
$\mathrm{M}$

$\mathrm{E}$

E

E

M 
PRICE SCALE FOR 1927

\begin{tabular}{|c|c|c|c|c|c|c|c|c|c|c|c|c|c|c|}
\hline \multicolumn{3}{|c|}{ Single Bulbs } & \multicolumn{3}{|c|}{10 Bulbs } & \multicolumn{3}{|c|}{25 Bulbs } & \multicolumn{3}{|c|}{50 Bulbs } & \multicolumn{3}{|c|}{100 Bulbs } \\
\hline 1 & 2 & 3 & 1 & 2 & 3 & 1 & 2 & 3 & 1 & 2 & 3 & 1 & 2 & 3 \\
\hline .05 & .04 & .03 & .40 & .32 & .24 & .88 & .70 & .53 & 1.50 & 1.20 & .90 & 2.50 & 2.00 & 1.50 \\
\hline .06 & .05 & .04 & .48 & .38 & .29 & 1.05 & .84 & .63 & 1.80 & 1.44 & 1.08 & 3.00 & 2.40 & 1.80 \\
\hline .07 & .06 & .04 & .56 & .45 & .31 & 1.23 & .98 & .74 & 2.10 & 1.68 & 1.26 & 3.50 & 2.80 & 2.10 \\
\hline .08 & .06 & .05 & .64 & .51 & .38 & 1.40 & 1.12 & .84 & 2.40 & 1.92 & 1.44 & 4.00 & 3.20 & 2.40 \\
\hline .09 & .07 & .05 & .72 & .58 & .43 & 1.58 & 1.26 & .95 & 2.70 & 2.16 & 1.62 & 4.50 & 3.60 & 2.70 \\
\hline .10 & .08 & .06 & .80 & .64 & .48 & 1.75 & 1.40 & 1.05 & 3.00 & 2.40 & 1.80 & 5.00 & 4.00 & 3.00 \\
\hline .12 & .10 & .07 & .96 & .77 & .58 & 2.10 & 1.68 & 1.26 & 3.60 & 2.88 & 2.16 & 6.00 & 4.80 & 3.60 \\
\hline .15 & .12 & .09 & 1.20 & .96 & .72 & 2.63 & 2.10 & 1.58 & 4.50 & 3.60 & 2.70 & 7.50 & 6.00 & 4.50 \\
\hline .18 & .14 & .11 & 1.44 & 1.15 & .86 & 3.15 & 2.52 & 1.89 & 5.40 & 4.32 & 3.21 & 9.00 & 7.20 & 5.40 \\
\hline .20 & .16 & .12 & 1.60 & 1.28 & .96 & 3.50 & 2.80 & 2.10 & 6.00 & 4.80 & 3.60 & 10.00 & 800 & 600 \\
\hline .25 & .20 & .15 & 2.00 & 1.60 & 1.20 & 4.38 & 3.50 & 2.63 & 7.50 & 6.00 & 4.50 & 12.50 & 10.00 & 7.50 \\
\hline .30 & .24 & .18 & 2.40 & 1.92 & 1.44 & 5.25 & 4.20 & 3.15 & 9.00 & 7.20 & 5.40 & 1500 & 12.00 & 9.00 \\
\hline .35 & .28 & .21 & 2.80 & 2.21 & 1.68 & 6.13 & 4.90 & 3.68 & 10.50 & 8.40 & 6.30 & 17.50 & 14.00 & 10.50 \\
\hline .40 & .32 & .24 & 3.20 & 2.56 & 1.92 & 7.00 & 5.60 & 4.20 & 12.00 & 960 & 7.20 & 20.00 & 1600 & 12.00 \\
\hline
\end{tabular}

DIRECTIONS

Find the line beginning with the first-size-single-bulb price shown on page 2 or 3 opposite the variety wanted. Follow that line toward the right to find p-ices for other sizes or quantities.

\section{BARGAIN COLLECTIONS}

10 Bulbs each of 10 varieties, 100 Bulbs in all, our selection, labeled and postpaid in the United States:

No. $1, \$ 3.50$

No. $2, \$ 2.80$

No. $3, \$ 2.10$

\section{DIRIGO MIXTURE}

Per 100, postpaid:

No. $1, \$ 2.25$

No. $2, \$ 1.80$

No. 3, \$1.35

\section{TERMS}

Cash with all orders. Please do not ask us to ship C. O. D., as this involves additional clerical work at a very busy season. Stock offered subject to prior sale. At prices shown herein, we prepay shipments to United States and Canadian points when order amounts to $\$ 1.00$ or more. On orders for less than $\$ 1.00$, add two cents per bulb. We have no more favorable terms, prices or discounts than shown in this catalogue.

\section{GUARANTEE}

We guarintee all bulus to be healthy, tiue to name and up to size; and will replace any found otherwise if claim is made during the present calendar year.

\section{SUBSTITUTIONS}

We never substitute without permission. When writing your order, please answer the following questions:

1st. Shall we substitute a similar variety of equal or greater value when stock of variety ordered is exhausted?

2nd. Shall we substitute equal value in another size of the same variety when stock of size ordered is exhausted?

We recommend early orders to avoid disappointment.

Address all communications:

JOHN E. SNELL

Dirigo Gladiolus Gardens

125 Concord Street

Portland, Maine 